\title{
Unit Cell Analysis of the Superelastic Behavior of Open-Cell Tetrakaidecahedral Shape Memory Alloy Foam under Quasi-Static Loading
}

\author{
Guillaume Maîtrejean, ${ }^{1,2}$ Patrick Terriault, ${ }^{1}$ Diego Devís Capilla, ${ }^{1}$ and Vladimir Brailovski ${ }^{1}$ \\ ${ }^{1}$ Department of Mechanical Engineering, École de technologie supérieure, Montréal, QC, Canada H3C 1K3 \\ ${ }^{2}$ Laboratoire Rhéologie et Procédés, 363 rue de la Chimie-Bâtiment B, Domaine Universitaire, BP 53, 38041 Grenoble Cedex 9, France \\ Correspondence should be addressed to Guillaume Maîtrejean; guillaume.maitrejean@ujf-grenoble.fr
}

Received 16 January 2014; Revised 2 April 2014; Accepted 18 April 2014; Published 8 July 2014

Academic Editor: Outi Söderberg

Copyright (C) 2014 Guillaume Maîtrejean et al. This is an open access article distributed under the Creative Commons Attribution License, which permits unrestricted use, distribution, and reproduction in any medium, provided the original work is properly cited.

Cellular solid materials and, more specifically, foams are increasingly common in many industrial applications due to their attractive characteristics. The tetrakaidecahedral foam microstructure, which can be observed in many types of foams, is studied in the present work in association with shape memory alloys (SMA) material. SMA foams are of particular interest as they associate both the shape memory effect and the superelasticity with the characteristics of foam. A Unit Cell Finite Element Method approach is used, an approach that allows accurate predicting of the macroscale response of the foam with a highly reduced numerical effort. The tetrakaidecahedral foam's responses, both in the elastic and in the superelastic stages, are then extracted and compared with results from the literature. The tetrakaidecahedral geometry is found to be of particular interest when associated with SMA as it takes more advantage of the superelastic property of the material than foams with randomly distributed porosity.

\section{Introduction}

Shape memory alloy materials are of major interest in a broad range of applications for their uncommon mechanical properties-shape memory effect and superelasticity $[1,2]$. Over the last decade, numerous studies have focused on the porous form of SMA, which combines the intrinsic assets of both SMA and foam materials. From an experimental point of view, SMA foams have been processed using different manufacturing techniques and a number of research papers have been published in various research fields, especially for biomedical applications (see, e.g., [3, 4] or, more recently, [5]). From a numerical point of view, intensive research has been conducted on both the determination of accurate constitutive models of dense SMA (a review of some models can be found in [6]) and the SMA foam modelling. Several studies have shown the importance of the foam's geometrical characteristics, that is, the size, shape, orientation, and distribution of the pore microstructure on its macroscale behavior [7].
Tetrakaidecahedral cellular solids are of interest to many researchers, since they were proven as far back as 1887 by Lord Kelvin [8] that they are the most efficient means to fill space within a given volume with a single shape; that is, they are the structure that provide the minimum surface area per given volume [7]. The tetrakaidecahedron, also called the Kelvin cell or the regular truncated octahedron, is characterized by 14 faces, eight regular hexagons and six squares, and can be generated by truncating the corners of a cube [9]. As depicted in Figure 1, a number of foams exhibit geometry which can be accurately modeled using a tetrakaidecahedron-based model. Even though such foams normally possess a very high porosity, it is now possible to obtain, using modern additive manufacturing methods, tetrakaidecahedral geometry-based foams with porosity ranging in very large limits, that is, from 1 to $99 \%$ of porosity $[10,11]$.

For these multiple reasons, the tetrakaidecahedral geometry of foam models has been widely studied in the literature 


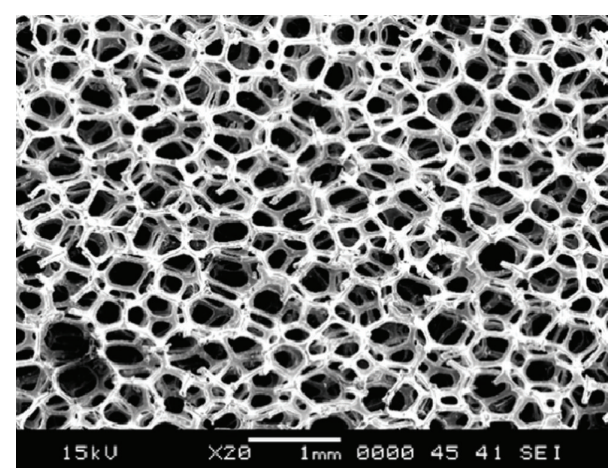

(a)

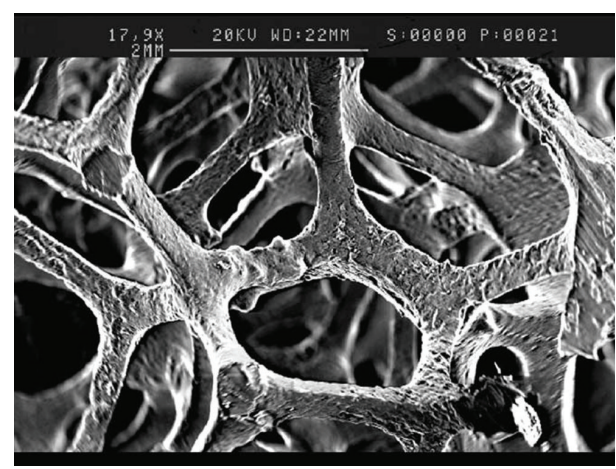

(b)

FIGURE 1: Tetrakaidecahedral-like metallic foam, from [12] (a) and from [13] (b).

from the analytical $[9,14,15]$, experimental [12], and numerical [16-18] perspectives.

In this study, we focus on the mechanical responses of tetrakaidecahedral open-cell SMA foam with a density ratio $\rho / \rho_{d}$ ranging from 0.01 to 1 , where $\rho$ denotes the density and the subscript $d$ is the fully dense value. Note that the terms porosity, density ratio, and pore volume fraction are synonyms and will alternatively and indistinctly be used in this work.

In particular, we aim to study the influence of the superelastic effect associated with open-cell tetrakaidecahedral foam. For this purpose, we define the model of tetrakaidecahedral foam from both a geometrical and a numerical point of view in Section 2. The tetrakaidecahedral foam responses in the elastic and in the superelastic stage are extracted in Section 3 and compared with results from the literature. We draw our conclusions in Section 4.

\section{Computational Setup}

2.1. SMA Constitutive Model. All the computations are performed using the commercial finite element software Ansys. The SMA model has already been implemented [19]. The model is briefly summarized below; the interested reader is invited to refer to [20] and the references therein for more information.

The material model considers the nonlinear behavior occurring during the reversible phase transformation from Austenite to Martensite or from Martensite to Austenite. For the sake of clarity, we adopt the following convention concerning exponents or subscripts: $A, M, A M$, and $M A$ will refer to Austenite, Martensite, Austenite to Martensite and Martensite to Austenite phase transformations, respectively. The Auricchio's model is able to model the tensioncompression asymmetry behavior but, as a first approximation, we do not account for such asymmetry in the present study. It is worth pointing out, however, that the asymmetry behavior has a great influence on the mechanical response of the model and the reader must keep in mind the assumption of a symmetric mechanical behavior.

It is assumed that the material behavior does not show any permanent strain and that the material is perfectly isotropic.
TABLE 1: Material parameters for dense SMA constitutive model.

\begin{tabular}{lc}
\hline Material parameters & Values \\
\hline$E_{A}(\mathrm{GPa})$ & 50 \\
$\nu$ & 0.3 \\
$\sigma_{s}^{A M}(\mathrm{MPa})$ & 150 \\
$\sigma_{f}^{A M}(\mathrm{MPa})$ & 200 \\
$\sigma_{s}^{M A}(\mathrm{MPa})$ & 100 \\
$\sigma_{f}^{M A}(\mathrm{MPa})$ & 50 \\
$\varepsilon_{L}$ & 0.01 \\
\hline
\end{tabular}

The following constants are used to model the SMA behavior and they are graphically represented in [21]:

(i) $\sigma_{s}^{A M}$ is the starting stress for the phase transformation from Austenite to Martensite;

(ii) $\sigma_{f}^{A M}$ is the final stress for the phase transformation from Austenite to Martensite;

(iii) $\sigma_{s}^{M A}$ is the starting stress for the phase transformation from Martensite to Austenite;

(iv) $\sigma_{f}^{M A}$ is the final stress for the phase transformation from Martensite to Austenite;

(v) $\varepsilon_{L}$ is the maximum superelastic strain;

(vi) $E_{A}$ is the Young's modulus of Austenite;

(vii) $E_{M}$ is the Young's modulus of Martensite;

(viii) $E_{A M}$ is the phase transformation modulus of austenite to Martensite;

(ix) $E_{M A}$ is the phase transformation modulus of Martensite to Austenite.

Throughout this paper, the porous SMA material will be defined by the parameters summarized in Table 1 . The associated stress-strain response for fully dense foam is depicted in Figure 2.

Note that to reduce the number of iterations required for a complete phase transformation, $\varepsilon_{L}$ is set to 0.01 , which is approximately $1 / 10$ of the $\mathrm{Ti}-\mathrm{Ni}$ superelastic range. 


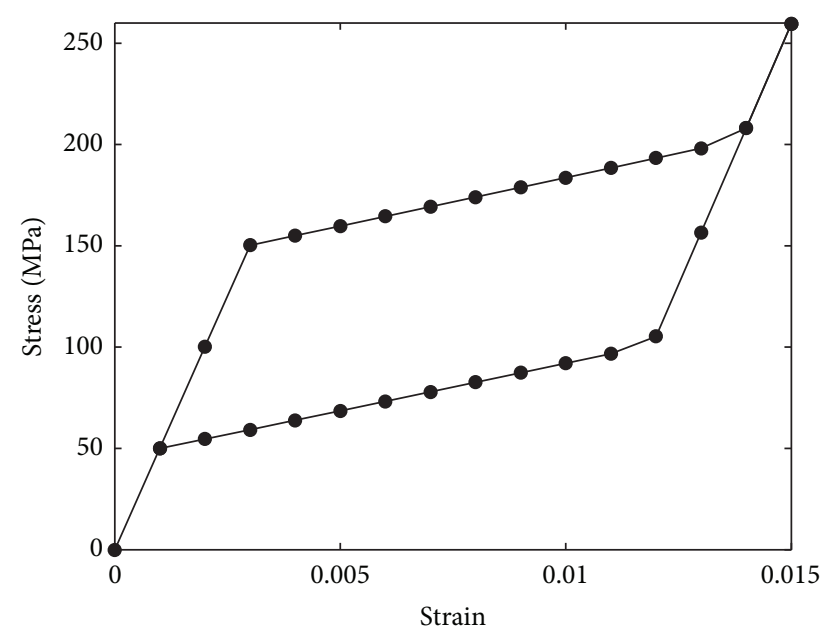

Figure 2: Stress-strain curve obtained for 0.015 maximum strain.

This scaling down does not affect the validity of the results obtained.

Note about the Model. The Auricchio model as implemented in Ansys software may be considered somewhat weak, as more sophisticated models have been published by different research groups $[23,24]$ since its appearance, even by the Auricchio's own research group [25]. However, this model presents two fundamental assets which make it very suitable for the purpose of the present work.

(i) Firstly, the model focuses on the superelastic behaviour of the SMA using only a few constitutive parameters, and it has been successfully validated in a number of studies.

(ii) Secondly, its direct implementation in the commercial software Ansys precludes the need to apply the USER Material Subroutine (USERMAT), thereby allowing the reader to easily verify the results obtained in this work.

In sum, in light of these assets, this model has been successfully applied in [21] as well as in [26] and the use of a more sophisticated model in this work is not justified.

\subsection{Unit Cell Finite Element Method Approach}

2.2.1. Geometrical Aspects. The geometrical model addressed in this work is based on an equisided tetrakaidecahedron which is defined by the side length $a$. The open-cell geometry is obtained from a fully dense tetrakaidecahedron by removing a similar, but smaller, tetrakaidecahedron volume from the inner space and by opening each of the 14 faces. The final tetrakaidecahedral geometry is depicted in Figure 3 in the global coordinate system $\left(\vec{X}_{g}, \vec{Y}_{g}, \vec{Z}_{g}\right)$ and the associated tetrakaidecahedral foam is obtained by repeating this geometry to fill space.

Due to the periodicity and symmetry exhibited by this foam, there is no need to reproduce a complete foam structure, since only a small part of it is needed to capture the macroscale behaviour of the foam, provided that the specific boundary conditions are applied (addressed in the next section). This approach, the so-called Unit Cell Finite Element Method (UCFEM), has already been widely used $[27,28]$ and makes it possible to circumvent the huge numerical weight exhibited by a full foam model.

The tetrakaidecahedral cell, that is, the UCFEM model associated with tetrakaidecahedral foam, is obtained by joining several tetrakaidecahedra together and performing appropriate sectioning. The final cell, depicted in Figure 4, is very similar to the one obtained by Zhu et al. [9] and Sullivan et al. [15], who performed reference works on tetrakaidecahedral cells via an analytical approach. Note that this cell possesses six symmetry planes and, consequently, the stress and the strain fields can be extended for the whole porous SMA. The stress is then the ratio of the total applied force to the area of the unit cell boundary. The strain along the loading path is the ratio of the displacement in the loading direction of the unit cell to the initial length of the unit cell.

However, contrary to Zhu et al. [9] and Sullivan et al. [15], who modeled the cell using beam elements (which limit the validity of the model for porosity less than 0.05 ), the present finite element model is meshed using brick elements (20 nodes) exhibiting a quadratic displacement behavior. A wider range of porosity variation can then be addressed.

2.2.2. Boundary Conditions. The boundary conditions are derived from the consideration of the symmetry conditions on the surfaces of the box enclosing the unit cell. Following the approach of [29], the reflection symmetries of the unit cell require that the shear stress vanishes at all points of the enclosing box and that the normal displacements on the surfaces are constant. A local coordinate system $(\vec{x}, \vec{y}, \vec{z})$ is then defined by the $x, y, z$ axes of the edges of the unit cell box (Figure 4), corresponding to the global coordinate system $\left(x_{g}, y_{g}, z_{g}\right)$ with a $\pi / 4$ rotation around the $z$-axis.

The displacements $(u, v, w)$ in this local coordinate system are given along $(\vec{x}, \vec{y}, \vec{z})$, respectively. In this framework, the displacement boundary conditions can be expressed by

$$
\begin{gathered}
u(0, y, z)=v(x, 0, z)=w(x, y, 0)=0, \\
u(2 a, y, z)=\bar{u}, \quad v(x, 2 a, z)=\bar{v}, \\
w(x, y,-\sqrt{2} a)=\bar{w}
\end{gathered}
$$

and the shear stress conditions by

$$
\begin{aligned}
\sigma_{x y}(0, y, z) & =\sigma_{x y}(x, 0, z)=\sigma_{x y}(2 a, y, z) \\
& =\sigma_{x y}(x, 2 a, z)=0, \\
\sigma_{x z}(0, y, z) & =\sigma_{x z}(x, y, 0)=\sigma_{x z}(2 a, y, z) \\
& =\sigma_{x z}(x, y,-\sqrt{2} a)=0, \\
\sigma_{y z}(x, 0, z) & =\sigma_{y z}(x, y, 0)=\sigma_{y z}(x, 2 a, z) \\
& =\sigma_{y z}(x, y,-\sqrt{2} a)=0,
\end{aligned}
$$




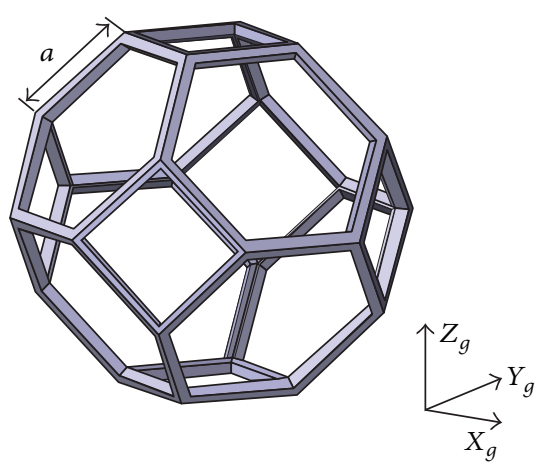

(a)

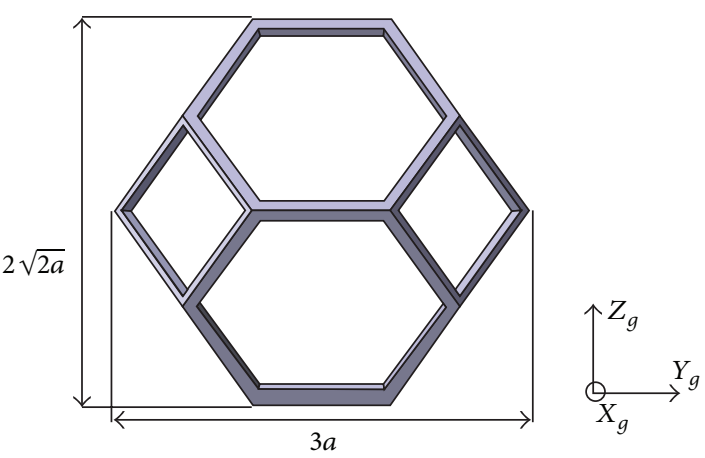

(b)

FIgURE 3: Equisided tetrakaidecahedron.

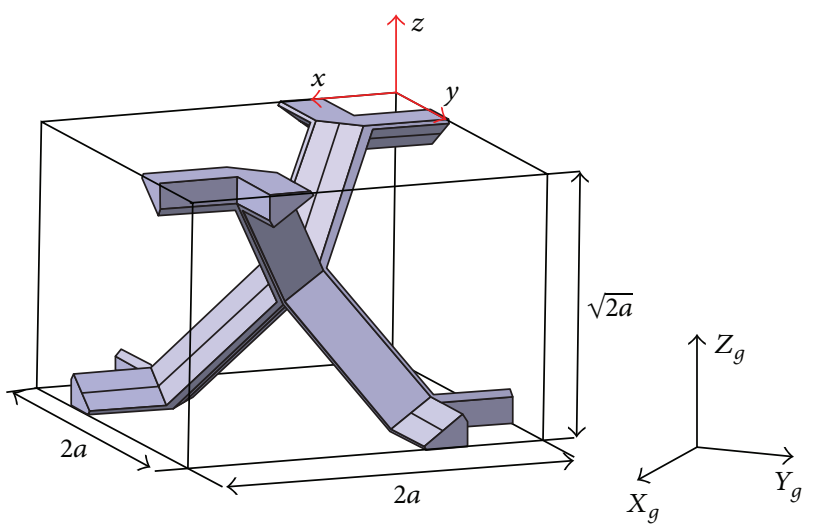

FIGURE 4: UCFEM model used for stress and strain calculations.

where - denotes the mean value, which imposes that all the material points on the respective unit cell boundaries have the same normal displacement.

The implementation of the displacement boundary conditions stated in (1) in Ansys software is straightforward, using coupled degrees of freedom. The shear stress boundary conditions given by (2), however, cannot be explicitly set in Ansys, but are implicitly defined using the kinematic boundary condition-defined (1). Figure 5 is a schematic illustration of these boundary conditions.

Finally, the finite element model of the Unit Cell is meshed using 20-nodes, mixed tetrahedral hexahedral, quadratic elements as depicted Figure 6.

This finite element model presents geometric singularities that may reduce the stress calculation reliability. To remove or, at least, to limit these risks, it is generally advised to round the edges and fillet the corners. However, that approach would lead to a more complex geometry, higher number of elements, and thus higher computational cost. For these reasons, the maximum stresses obtained with our model are definitely overestimated.

2.2.3. Simplifications. When experiencing high strains, tetrakaidecahedral foam is subjected to highly nonlinear effects such as cracks or nonlinear buckling that may eventually

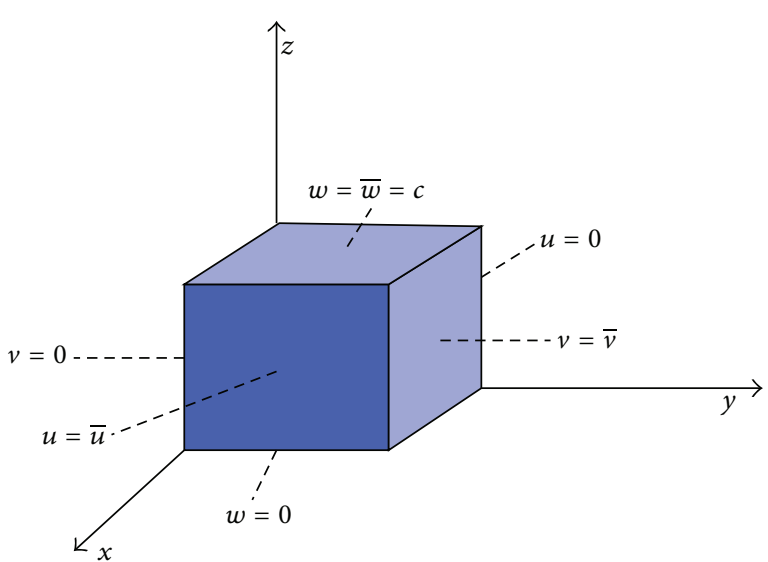

FIGURE 5: Schematic boundary conditions where $(u, v, w)$ are the displacements, - denotes the mean value, and $c$ is a homogeneous displacement of the upper face.

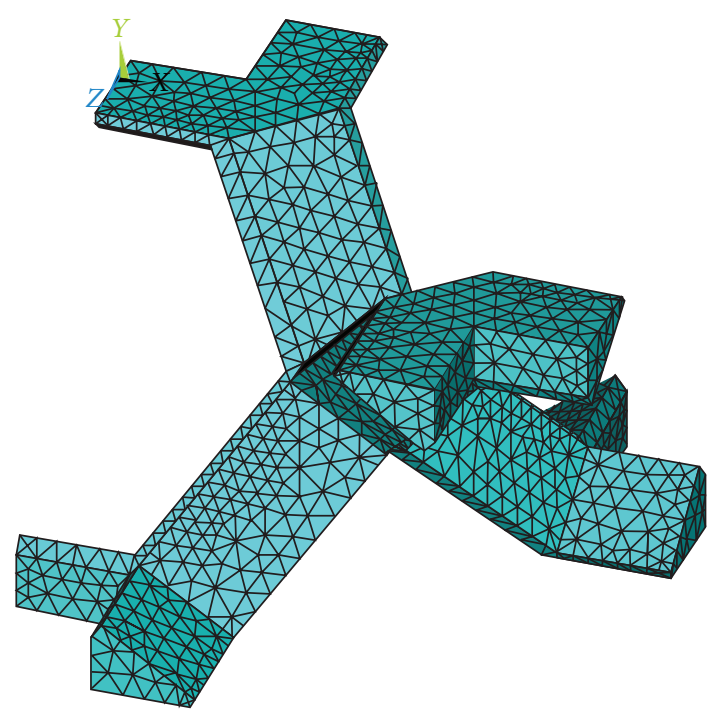

FIgUre 6: Mesh of the finite element model for the $\rho / \rho_{d}=0.1$ unit cell porosity. 


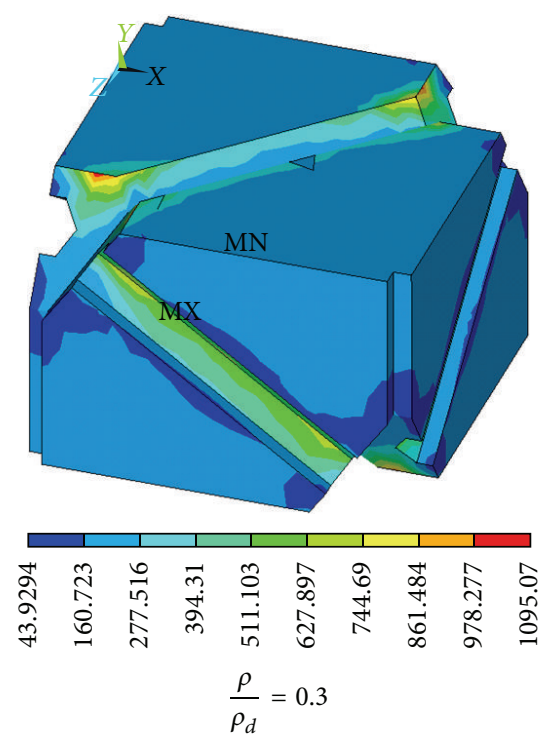

(a)

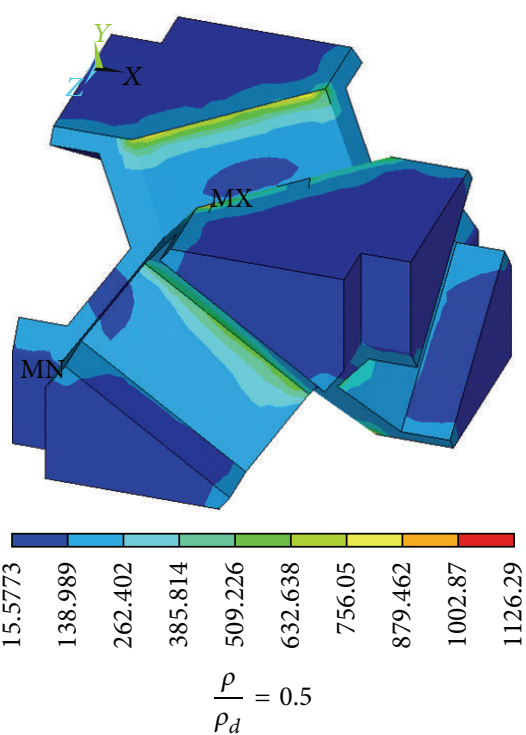

(b)

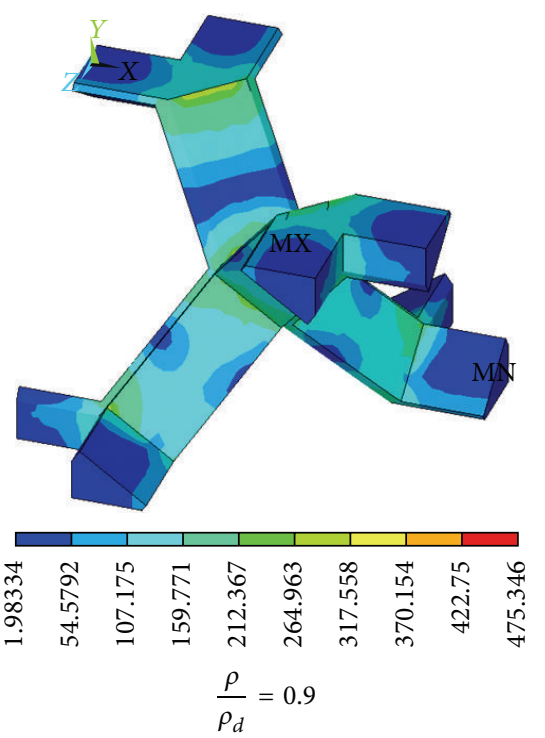

(c)

FIGURE 7: Illustration of microscale response with the Von-Mises stress of a unit cell in compression $(\varepsilon=0.015)$ during the loading stage for different porosities.

result either in a collapse or a destruction of the cell under compressive or tensile loading, respectively. However, the present work aims at studying the superelastic response of tetrakaidecahedral foam and, for this reason, only linearelastic and superelastic behaviours are addressed here.

\section{Results: Superelastic Behaviour}

The mechanical response of tetrakaidecahedral foam can be observed either at a micro- or a macroscale, that is, by looking at the response at the cell scale or at the overall sample scale. Most of the experimental results that can be found in the literature are naturally limited to the macroscale given difficulties obtaining this information at the microscale. Fortunately, we can illustrate both scales by using numerical approaches, and we address both perspectives in the following subsections.

\subsection{Microscale Response. Figure 7 illustrates the stress state in} a unit cell for a maximum compressive strain of $\varepsilon=0.015$.

As one can expect, we observe high stress heterogeneity with stress concentration due to the sharp edges of the geometry.

In order to see how the phase transformation evolves during loading, we depict, in Figures 8(a) and 8(b), the volume fraction of material having an equivalent stress $\sigma_{\text {eq }}$ greater than $\sigma_{s}^{A M}$ and $\sigma_{f}^{A M}$ as functions of applied strain. In both figures, we can observe that for all porosity foams, the higher the density, the higher the volume fractions of the material undergoing phase transformation and being fully transformed, respectively, in Figure 8(a) and in Figure 8(b) (which corresponds to a volume fraction of 1). These observations support those made for random SMA foams [21, 22] that martensitic transformation will never be completed in porous SMAs.

3.2. Macroscale Response. Stress-strain curves from both tension and compression tests are depicted in Figure 9 for various porosities. We can observe the large range of stresses exhibited by the samples of different porosity (more than three orders of magnitude). It has been shown for random foam $[21,22]$ that, for high porosity fraction, a full martensitic transformation can never be reached, even at very large strain. To confirm this conclusion, the maximum applied macroscale strain is set to 0.03 , which is three times the $\varepsilon_{L}$ (see Table 1). As expected, from $\rho / \rho_{d}>0.3$ to higher porosities, $\sigma_{f}^{A M}$ and $\sigma_{s}^{M A}$ stresses are never reached. Furthermore in this work, no difference is assumed between compression and tension in the Ansys SMA constitutive model. The very close responses between the compression and tension tests of Figure 9 indicate that the behavior of all the foams tested is not affected by the load direction, and, therefore, only tensile results could be addressed hereafter.

Figure 10 depicts the evolution of the constitutive model parameters ratio of, respectively, $E_{A}, E_{A M}, E_{M}, E_{M A}$, and $\sigma_{s}^{A M}$ and $\sigma_{f}^{M A}$ as functions of porosity. By "ratio" of any parameter we denote the apparent value corresponding to a given porosity divided by the fully dense value. We observe that the ratios are almost identical for critical stresses (Figure 10(a)), as well as for the moduli (Figure 10(b)), even if $E_{M}$ shows a slight offset.

The figures are scaled logarithmically, meaning that the slope of the plots corresponds to the exponent $n$ of a power law function equation $y=c x^{n}$. As observed experimentally in [30] and numerically in [16], the exponent $n$ is not constant over the whole porosity fraction range. We observe three 

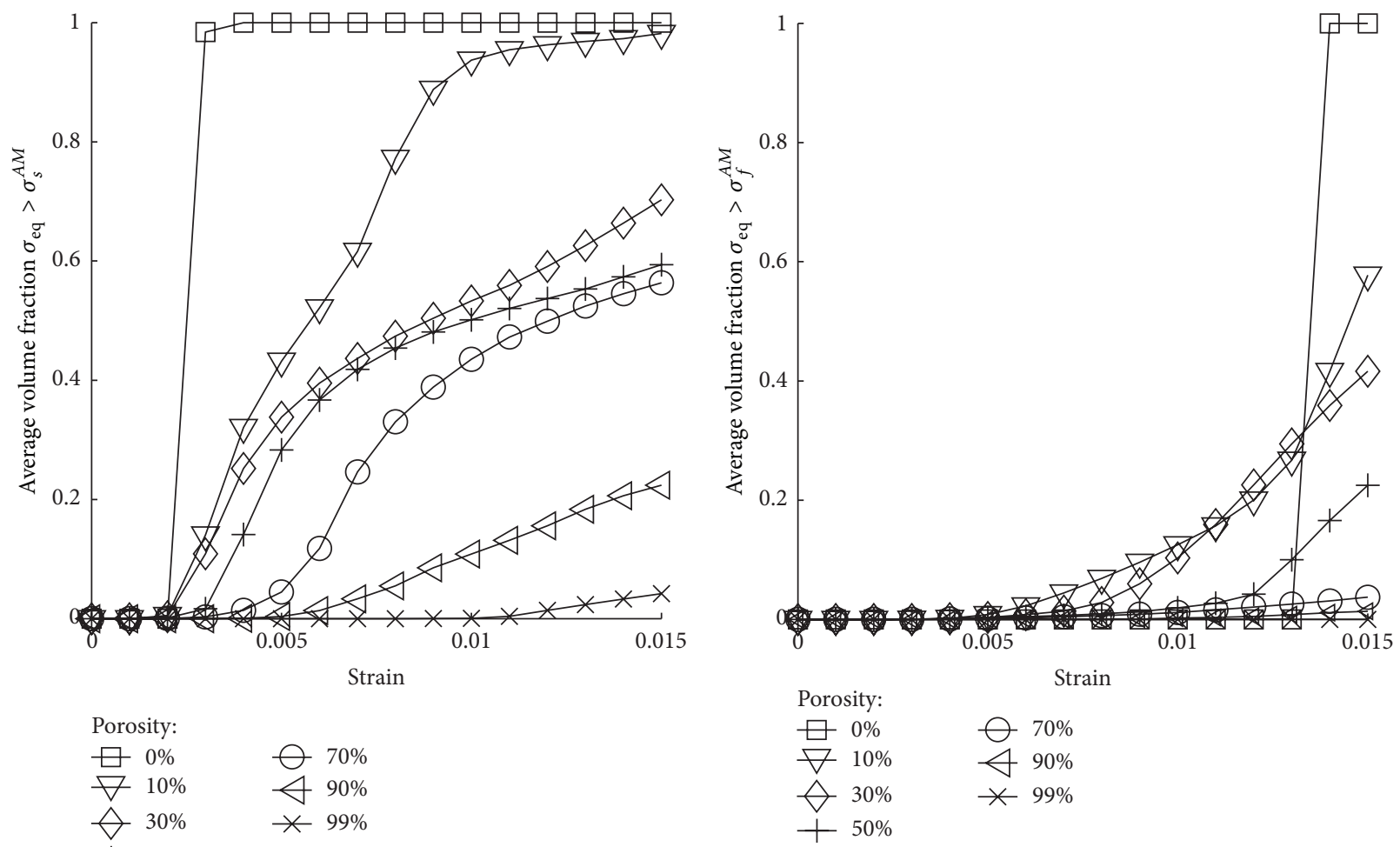

(a)

(b)

FIGURE 8: Volume fraction of the models exhibiting an equivalent stress $\sigma_{\text {eq }}$ greater than the phase transformation stress $\sigma_{s}^{A M}$ (a) and greater than the phase transformation stress $\sigma_{f}^{A M}(\mathrm{~b})$ as a function of the applied strain.

different regions, corresponding to high porosity $\left(\rho / \rho_{d}<\right.$ $0.25)$, medium porosity $\left(0.25<\rho / \rho_{d}<0.8\right)$, and low porosity $\left(\rho / \rho_{d}>0.8\right)$ fractions.

\section{Discussion}

4.1. Superelastic Response. We compare, in Figure 11, the volume fractions of the material undergoing phase transformation (from Figure 8) with the ones obtained for random foam of same porosity [21]. Significant differences between the regular and random foams' susceptibility to phase transformation can be observed as follows.

(1) For low porosities (Figure 11(a)), the volume fraction of material for which the phase transformation is initiated $\left(\sigma_{\mathrm{eq}}>\sigma_{s}^{A M}\right)$ for tetrakaidecahedral foam is far less notable than for random foam. The same trend is also observed for the volume fraction of fully transformed material $\left(\sigma_{\mathrm{eq}}>\sigma_{f}^{A M}\right)$.

(2) For moderate porosities (Figure 11(b)), the volume fraction of material, under transformation or fully transformed, follows a similar trend until a macroscale strain of 0.0075 . For larger strains, the phase transformation in the tetrakaidecahedral foam is less than in the random foam.
(3) Finally, for high porosities (Figure 11(c)), we observe two different trends. On one hand, the fractions of fully transformed material are almost equal for both geometries. On the other hand, the volume fraction of material undergoing $A \rightarrow M$ transformation is far more significant for the tetrakaidecahedral foam, but only when strain exceeds 0.005 .

Note that these conclusions are deemed valid despite the slight differences in the threshold phase transformation strains used in these works: $\varepsilon_{s}^{M A}=0.003$ and $\varepsilon_{f}^{M A}=0.013$ (this work) and $\varepsilon_{s}^{M A}=0.0025$ and $\varepsilon_{f}^{M A}=0.0125$ [21].

The differences revealed in the results indicate that tetrakaidecahedral foam will exhibit better superelastic behaviour than random foam, in accordance with the following observations.

(i) For low-porosity (Figure 11(a)), the periodic distribution and the pore shape regularity of tetrakaidecahedral foams lead to a decrease of the material volume fraction that is fully transformed. This is an interesting feature, since a lesser proportion of such a material will ultimately reach the yield stress, generate irreversible strains, and lead to fracture.

(ii) For high-porosity (Figure 11(c)), the periodic pore structure tends to drastically increase the amount 


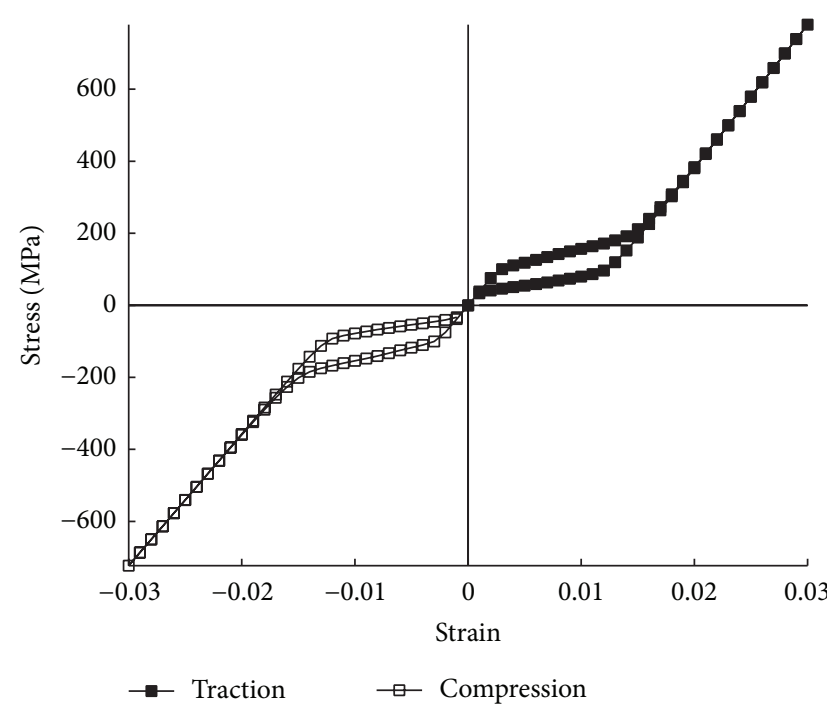

(a) $\rho / \rho_{d}=0.9$

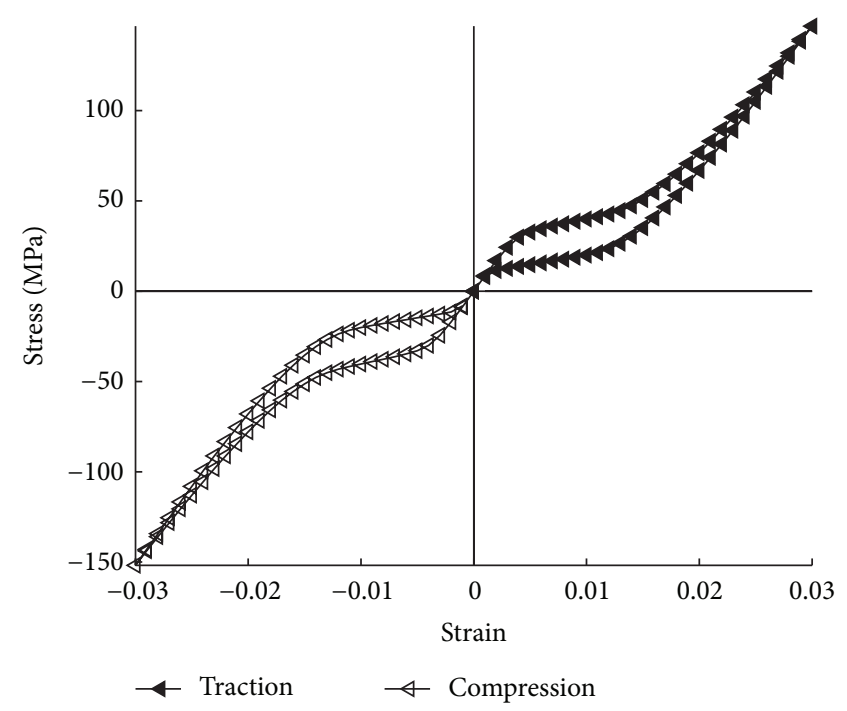

(c) $\rho / \rho_{d}=0.5$

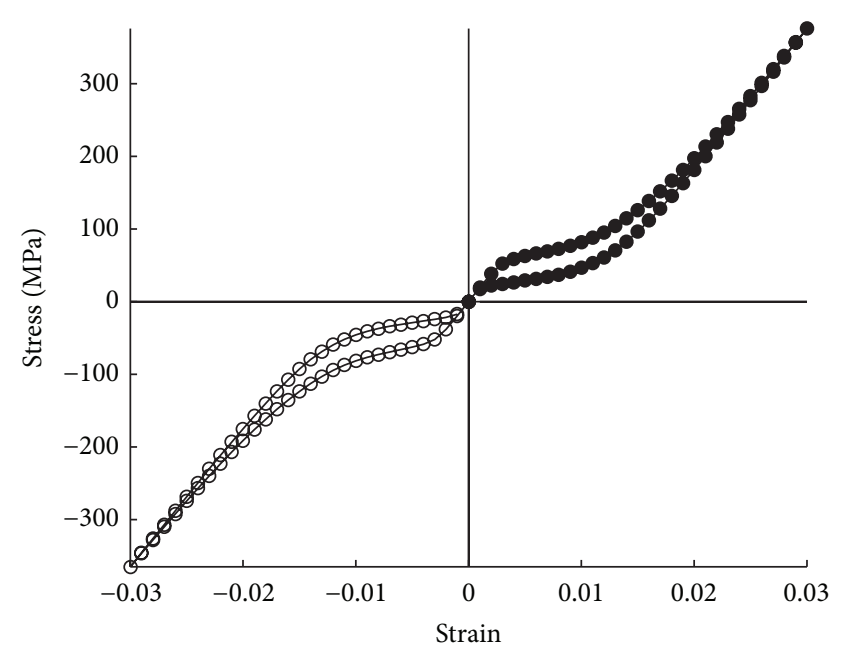

$\rightarrow$ Traction $\quad \multimap$ Compression

(b) $\rho / \rho_{d}=0.7$

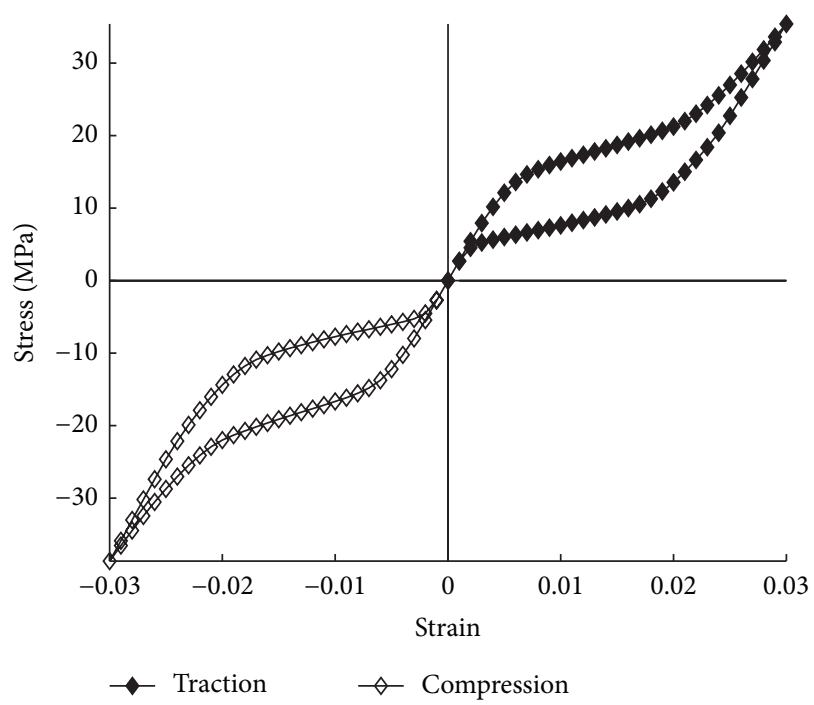

(d) $\rho / \rho_{d}=0.3$

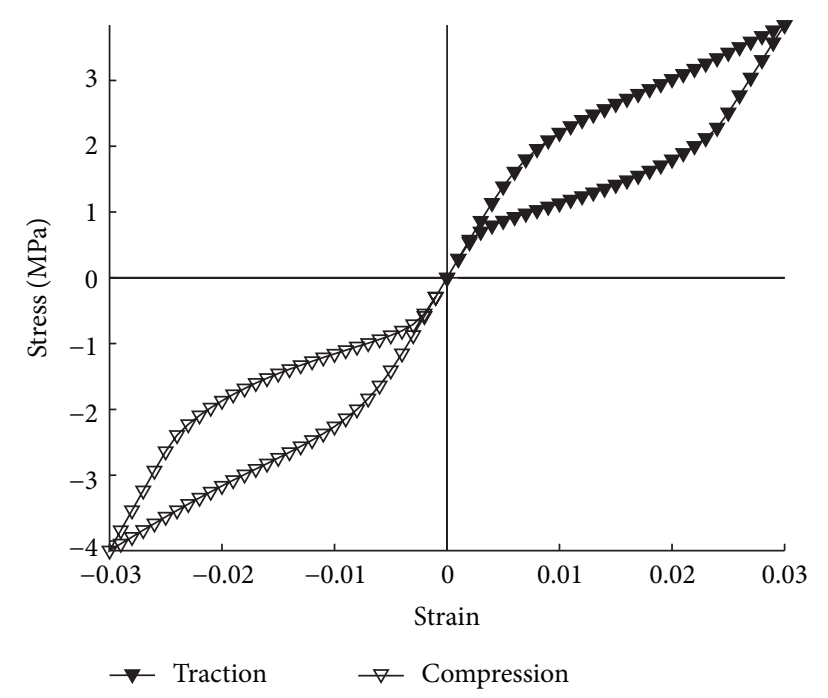

(e) $\rho / \rho_{d}=0.1$

FIGURE 9: Macroscale stress-strain responses for several porosities. 


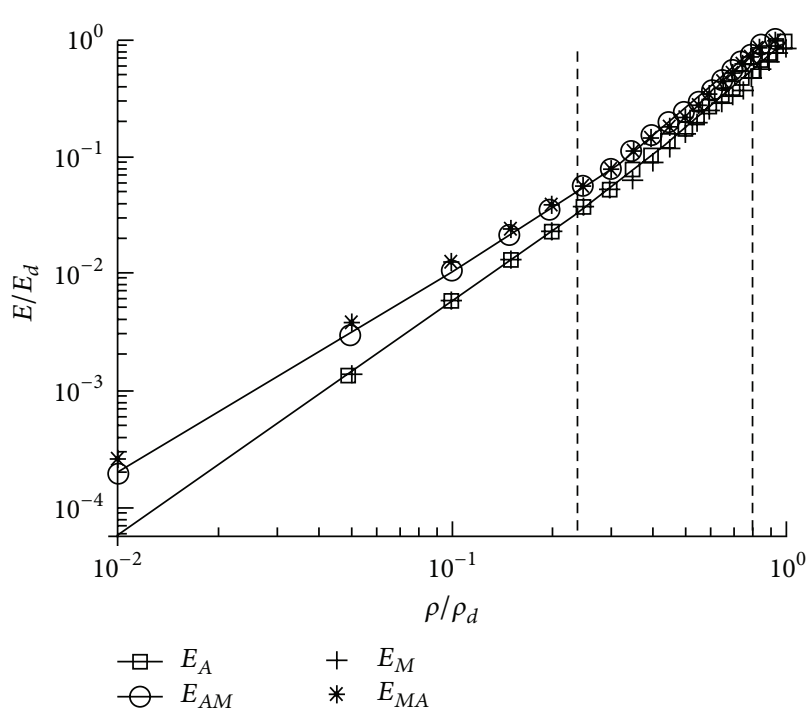

(a) Elastic moduli

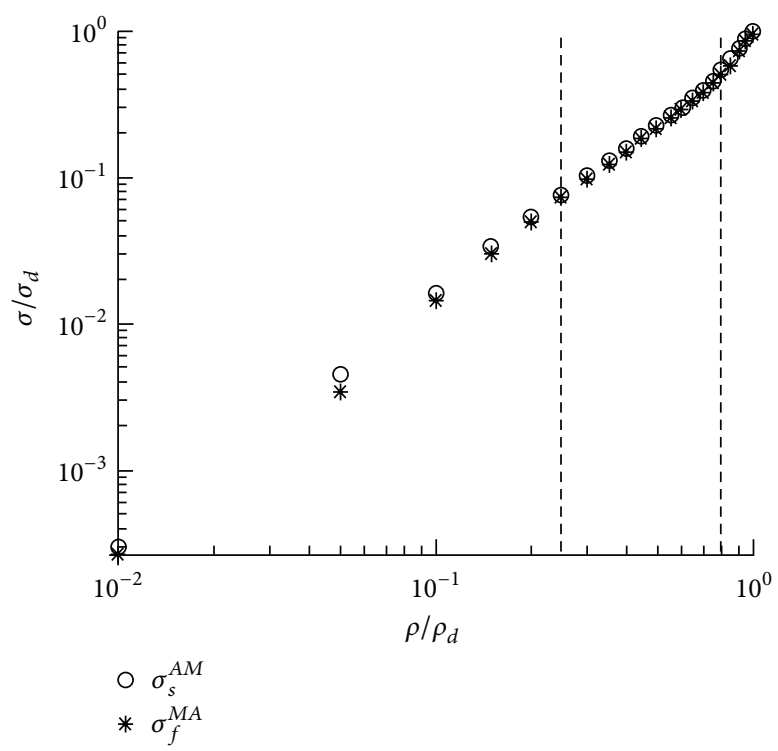

(b) Critical stresses

FigURE 10: Evolution of the constitutive model parameters as a function of porosity fraction: (a) $E_{A}, E_{A M}, E_{M}$, and $E_{M A}$; (b) $\sigma_{s}^{A M}$ and $\sigma_{f}^{M A}$.

of material undergoing phase transformation, while keeping the completely transformed material fraction unchanged. With a larger proportion of the material participating in the phase transformation, the macroscopic strain is better distributed throughout the entire sample.

4.2. Apparent Elastic Modulus. In the global coordinate system in which the tetrakaidecahedral geometry has been defined, the model is isotropic along the principal axes. The apparent elastic modulus ratio of the unit cell, that is, the ratio of the apparent elastic modulus of the unit cell and of the fully dense material, is depicted in Figure 12 for both traction and compression tests at different porosity fractions and compared to results from the literature.

We first observe that the compression and tension results are perfectly superimposed, meaning that no difference emerges between these two loadings as far as it concerns apparent elastic modulus.

Next, when comparing with the well-known Gibson and Ashby cellular solid equation of the evolution of the elastic modulus, that is, $E / E_{d}=C\left(\rho / \rho_{d}\right)^{2}$ (with $C=1$ generally used), we observe that the evolution is parallel to that of the present work from very high $\left(\rho / \rho_{d}=0.01\right)$ to moderately low $\left(\rho / \rho_{d}=0.7\right)$ porosities. For lower porosities, the tetrakaidecahedral elastic modulus ratio converges to the fully dense value fraction, $E / E_{d}=1$. The analytical results from [9] for tetrakaidecahedral foams of high porosity, based on beam theory, are in very good agreement with our tetrakaidecahedral cells modeled using circular beam elements. The elastic moduli of random pores calculated by Roberts and Garboczi [16] are in perfect agreement with the Gibson and Ahsby's predictions, and, thus, their evolution is parallel to that of the tetrakaidecahedral foam.
Finally, the results from [21], which depict the evolution of the elastic moduli for SMA foams with random pores (random shape, size, and distribution), produce a trend close to that of Gibson and Ashby, with a slight offset to the present results.

4.3. Stress Concentration Factor. Following the definition of the stress concentration factor introduced by Panico and Brinson [22] in the apparent elastic regime as being the ratio of the maximum axial stress component to the applied macroscopic stress, Figure 13 depicts the stress concentration factor for tetrakaidecahedron cell and random foams [22].

As expected, the concentration factor obtained in the present work follows the same trend as the factor from random foams: the higher the porosity, the higher the concentration factor. However, for the low-porosity fraction, the stress concentration factor of the tetrakaidecahedral cell is lower (Figure 13 is a log-log plot) than the factors from [22], which indicates that the periodic foam studied here will exhibit an increased fatigue strength than Panico's random foams.

With the geometrical models used in this work, a large amount of sharp edges are obtained. Local stress concentrations will then be inevitably generated, but it is not obvious that a significant influence would be observed at the macroscale level. To address this concern, other similar simulations should be carried out by rounding the sharp edges or by building the tetrakaidecahedral unit cell with circular extrusions.

4.4. Linear Buckling. The analysis of linear buckling in tetrakaidecahedral foam cells has been thoroughly investigated in [14], based on the beam theory developed by Timoshenko 


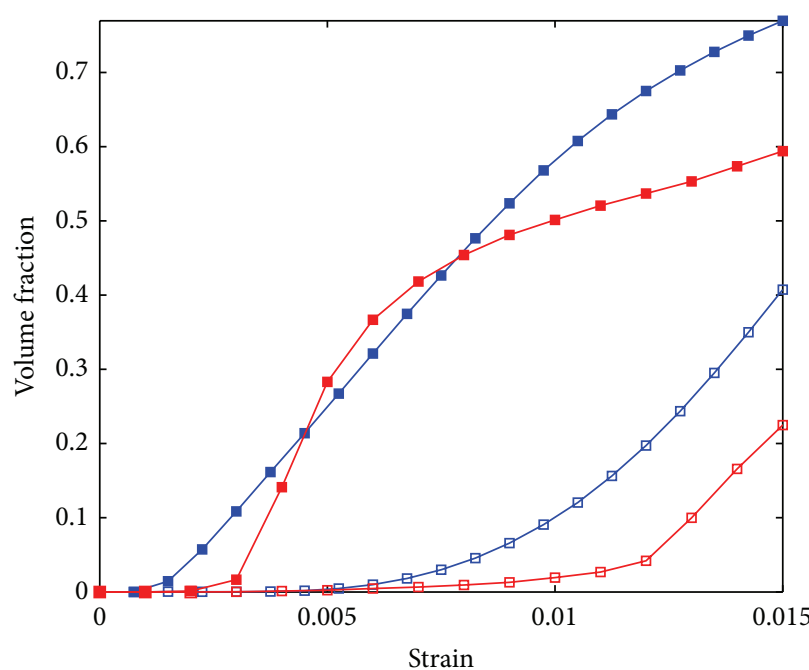

$-\sigma_{\text {eq }}>\sigma_{s}^{A M}$, Maîtrejean et al. $\rightarrow \sigma_{\text {eq }}>\sigma_{s}^{A M}$, present work $\rightarrow \sigma_{\text {eq }}>\sigma_{f}^{A M}$, Maîtrejean et al. $\rightarrow \sigma_{\text {eq }}>\sigma_{f}^{A M}$, present work

(a) $\mathrm{PVF}=0.3$

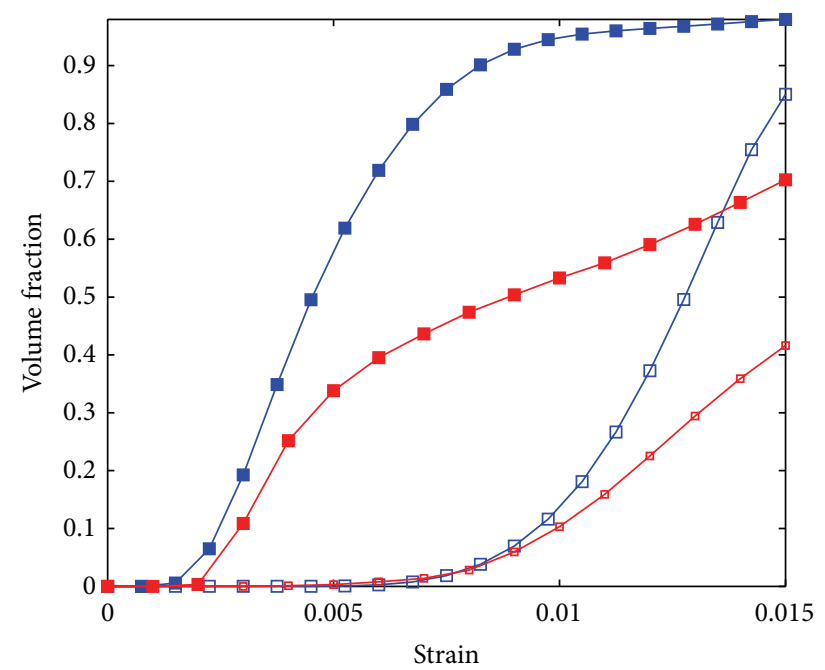

$-\sigma_{\mathrm{eq}}=\sigma_{s}^{A M}$, Maîtrejean et al. $-\sigma_{\mathrm{eq}}=\sigma_{s}^{A M}$, present work $\square \sigma_{\mathrm{eq}}=\sigma_{f}^{A M}$, Maîtrejean et al. $\rightarrow-\sigma_{\mathrm{eq}}=\sigma_{f}^{A M}$, present work

(b) $\mathrm{PVF}=0.5$

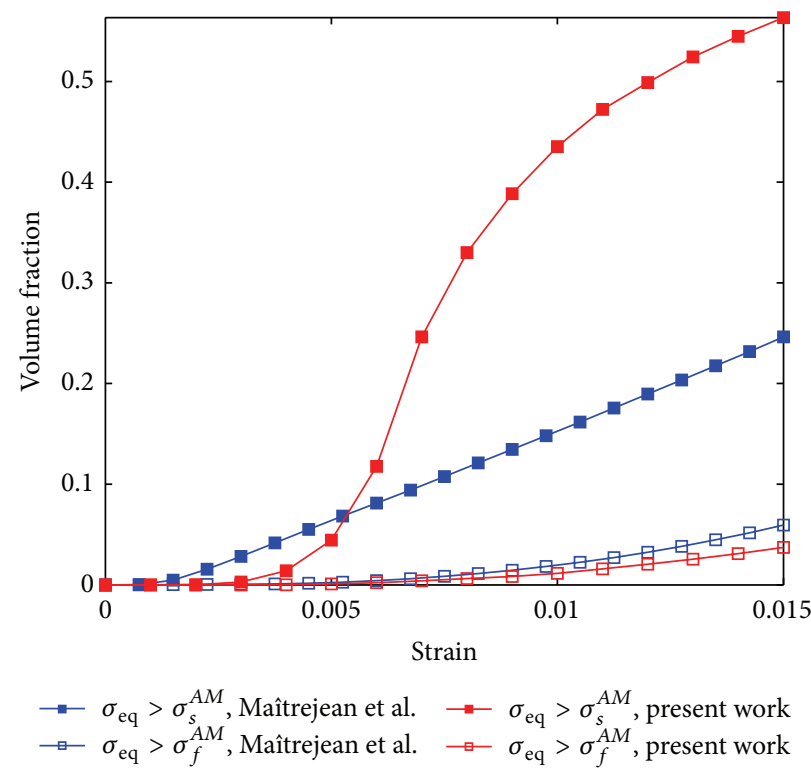

(c) $\mathrm{PVF}=0.7$

FIGURE 11: Average volume fraction of phases in the model as a function of strain for three different pore volume fractions: $(\mathrm{a})$ PVF $=0.3$, (b) $\mathrm{PVF}=0.5$, and (c) PVF $=0.7$ in highly-random pore foam, from [21], and in tetrakaidecahedral foam. The volume fraction that is experiencing phase transformation is highlighted.

and Gere [31]. It is assumed that the edges of the geometry are long thin beams; that is, that the relative density of the foam is low. Zhu et al. [14] showed that, for polymeric foam exhibiting viscoplastic behaviour, buckling was unlikely to happen. A more general approach by Gibson and Ashby [7], based on experimental elastic collapse, gives the onset buckling stress as follows:

$$
\sigma_{\mathrm{el}}^{*} \approx 0.03 E\left(\frac{\rho}{\rho_{d}}\right)^{2}\left(1+\left(\frac{\rho}{\rho_{d}}\right)^{1 / 2}\right)^{2} .
$$

In Figure 14, by comparing the predicted onset buckling stress from (3) with the phase transformation stress $\sigma_{s}^{A M}$ of the material, we can see that this geometry is not subjected to linear buckling for all the density fractions, as the yield stress reached is at least one order of magnitude lower. Furthermore, in light of these results, it is more than likely that the tetrakaidecahedral foam will experience plastic deformation and cracking before being subjected to any nonlinear buckling effect. 


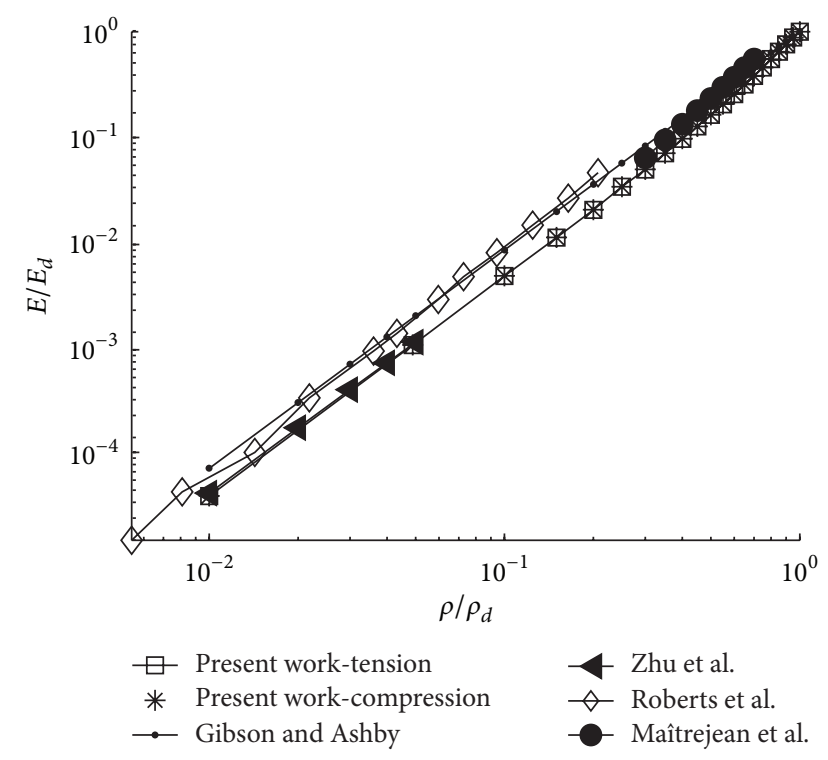

FIGURE 12: Comparison of the evolution of the apparent elastic modulus between the present work (compression and tension) and results from $[7,9,16,21]$.

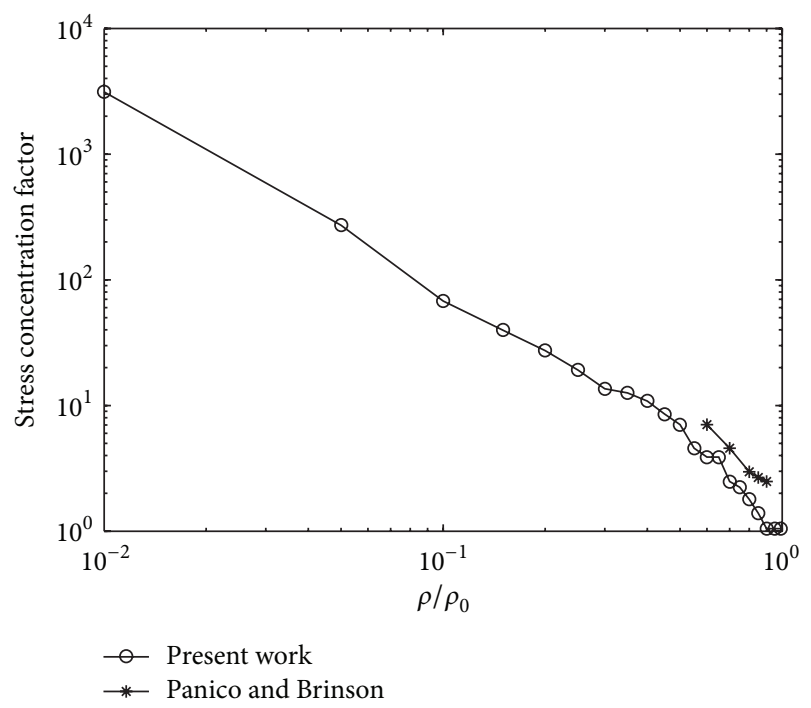

FIGURE 13: Stress concentration factor as a function of porosity for both tetrakaidecahedral and random foam from [22].

\section{Conclusion}

This work was designed to gain a deeper insight of the mechanical response of superelastic SMA foam based on open-cell tetrakaidecahedral geometry. Utilizing the UCFEM approach, a finite element model has been defined to accurately study the complete porosity fraction, from fully dense to its highest-porosity limit, that is, to as much as $99 \%$ of empty space. The numerical responses were obtained by using a simple but, from a numerical point of view, very effective SMA constitutive model in the Ansys environment. Numerous results were then extracted in the elastic regime

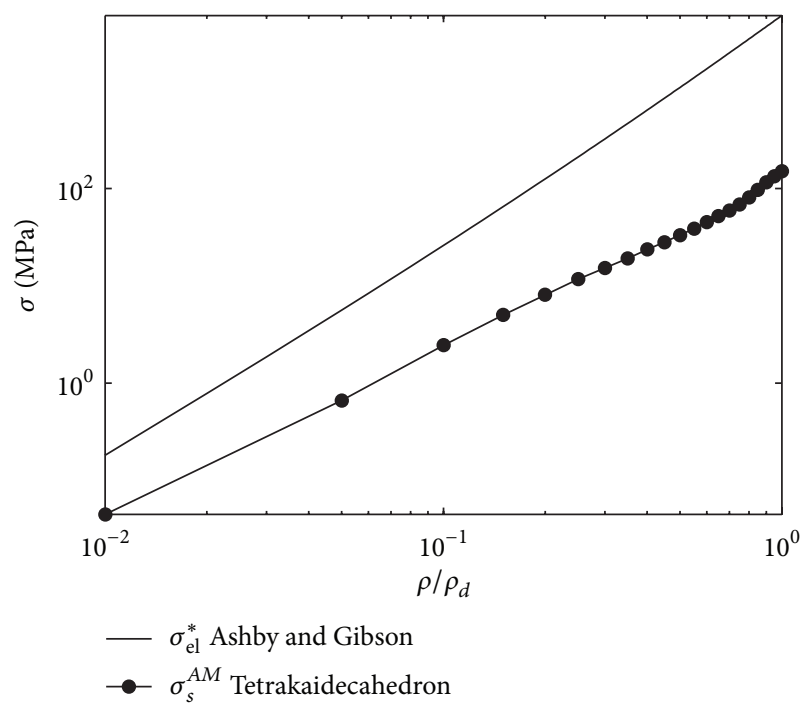

FIGURE 14: Evolution of both the onset buckling stress and the yield stress $\sigma_{s}^{A M}$ as a function of the density ratio.

as well as in the superelastic regime for the whole range of porosity.

Furthermore, it was observed that, due to the regular spatial distribution and shape of the pores in the tetrakaidecahedral foam, we can expect random foam to be stiffer than periodic foam with moderately-to-low porosity. On the contrary, for high porosities, we would expect periodic foam to be stiffer than random foam.

Finally, we have seen, both in the elastic regime through the stress factor and in the inelastic regime, by comparing the volume fraction of phase transformed material, that the tetrakaidecahedral geometry provides an obvious synergetic effect when coupled with superelasticity. For example, when comparing regular and random foams in the 0.5 to 0.99 porosity range, both structures show similar quantity of the completely transformed material, while the fraction of material undergoing martensitic transformation is much larger in the regular than in the random foams, making the load sharing much more efficient.

\section{Conflict of Interests}

The authors declare that there is no conflict of interests regarding the publication of this paper.

\section{References}

[1] V. Brailovski, S. Prokoshkin, P. Terriault, and F. Trochu, Shape Memory Alloys: Fundamentals, Modeling and Applications, École de Technologie Supérieure, 2003.

[2] D. C. Lagoudas, Shape Memory Alloys: Modeling and Engineering Applications, Springer, New York, NY, USA, 2008.

[3] Y. Zhao, M. Taya, Y. Kang, and A. Kawasaki, "Compression behavior of porous NiTi shape memory alloy," Acta Materialia, vol. 53, no. 2, pp. 337-343, 2005. 
[4] J. Y. Xiong, Y. C. Li, X. J. Wang, P. D. Hodgson, and C. E. Wen, "Titanium-nickel shape memory alloy foams for bone tissue engineering," Journal of the Mechanical Behavior of Biomedical Materials, vol. 1, no. 3, pp. 269-273, 2008.

[5] V. Brailovski, S. Prokoshkin, M. Gauthier et al., "Bulk and porous metastable beta $\mathrm{Ti}-\mathrm{Nb}-\mathrm{Zr}(\mathrm{Ta})$ alloys for biomedical applications," Materials Science and Engineering C, vol. 31, no. 3, pp. 643-657, 2011.

[6] V. Birman, "Review of mechanics of shape memory alloy structures," Applied Mechanics Reviews, vol. 50, no. 11, pp. 629-645, 1997.

[7] L. J. Gibson and F. M. Ashby, Cellular Solids: Structure and Properties, Cambridge University Press, 1999.

[8] L. Kelvin, "Stability of fluid motion: rectilinear motion of viscous fluid between two parallel plates," Philosophical Magazine, vol. 24, no. 5, pp. 188-196, 1887.

[9] H. X. Zhu, J. F. Knott, and N. J. Mills, "Analysis of the elastic properties of open-cell foams with tetrakaidecahedral cells," Journal of the Mechanics and Physics of Solids, vol. 45, no. 3, pp. 319-343, 1997.

[10] M. C. Melican, M. C. Zimmerman, M. S. Dhillon, A. R. Ponnambalam, A. Curodeau, and J. R. Parsons, "Three-dimensional printing and porous metallic surfaces: a new orthopedic application," Journal of Biomedical Materials Research, vol. 55, no. 2, pp. 194-202, 2001.

[11] G. Ryan, A. Pandit, and D. P. Apatsidis, "Fabrication methods of porous metals for use in orthopaedic applications," Biomaterials, vol. 27, no. 13, pp. 2651-2670, 2006.

[12] N. Michailidis, F. Stergioudi, H. Omar, and D. N. Tsipas, "Investigation of the mechanical behavior of open-cell Ni foams by experimental and FEM procedures," Advanced Engineering Materials, vol. 10, no. 12, pp. 1122-1126, 2008.

[13] Y. W. Kwon, R. E. Cooke, and C. Park, "Representative unit-cell models for open-cell metal foams with or without elastic filler," Materials Science and Engineering A, vol. 343, no. 1-2, pp. 63-70, 2003.

[14] H. X. Zhu, N. J. Mills, and J. F. Knott, "Analysis of the high strain compression of open-cell foams," Journal of the Mechanics and Physics of Solids, vol. 45, no. 11-12, pp. 1875-1904, 1997.

[15] R. M. Sullivan, L. J. Ghosn, and B. A. Lerch, "A general tetrakaidecahedron model for open-celled foams," International Journal of Solids and Structures, vol. 45, no. 6, pp. 1754-1765, 2008.

[16] A. P. Roberts and E. J. Garboczi, "Elastic properties of model random three-dimensional open-cell solids," Journal of the Mechanics and Physics of Solids, vol. 50, no. 1, pp. 33-55, 2002.

[17] S. K. Nammi, P. Myler, and G. Edwards, "Finite element analysis of closed-cell aluminium foam under quasi-static loading," Materials and Design, vol. 31, no. 2, pp. 712-722, 2010.

[18] P. Thiyagasundaram, J. Wang, B. V. Sankar, and N. K. Arakere, "Fracture toughness of foams with tetrakaidecahedral unit cells using finite element based micromechanics," Engineering Fracture Mechanics, vol. 78, no. 6, pp. 1277-1288, 2011.

[19] I. Ansys, Ansys Mechanical APDL and Mechanical Applications Theory Reference, Ansys, 2010.

[20] F. Auricchio, R. L. Taylor, and J. Lubliner, "Shape-memory alloys: macromodelling and numerical simulations of the superelastic behavior," Computer Methods in Applied Mechanics and Engineering, vol. 146, no. 3-4, pp. 281-312, 1997.

[21] G. Maîtrejean, P. Terriault, and V. Brailovski, "Density dependence of the superelastic behavior of porous shape memory alloys: representative volume element and scaling relation approaches," Computational Materials Science, vol. 77, pp. 93101, 2013.

[22] M. Panico and L. C. Brinson, "Computational modeling of porous shape memory alloys," International Journal of Solids and Structures, vol. 45, no. 21, pp. 5613-5626, 2008.

[23] D. C. Lagoudas and P. B. Entchev, "Modeling of transformationinduced plasticity and its effect on the behavior of porous shape memory alloys-part I: constitutive model for fully dense SMAs," Mechanics of Materials, vol. 36, no. 9, pp. 865-892, 2004.

[24] M. Panico and L. C. Brinson, "A three-dimensional phenomenological model for martensite reorientation in shape memory alloys," Journal of the Mechanics and Physics of Solids, vol. 55, no. 11, pp. 2491-2511, 2007.

[25] F. Auricchio, A. Reali, and U. Stefanelli, "A three-dimensional model describing stress-induced solid phase transformation with permanent inelasticity," International Journal of Plasticity, vol. 23, no. 2, pp. 207-226, 2007.

[26] G. Mastrejean, P. Terriault, V. Brailovski, and G. Maîtrejean, "Density dependence of the macroscale superelastic behavior of porous shape memory alloys: a two-dimensional approach," Smart Materials Research, vol. 2013, Article ID 749296, 13 pages, 2013.

[27] S. Nemat-Nasser and M. Hori, Micromechanics, Overall Properties of Heterogeneous Materials, Elsevier, Amsterdam, The Netherlands, 1999.

[28] M. A. Qidwai, P. B. Entchev, D. C. Lagoudas, and V. G. DeGiorgi, "Modeling of the thermomechanical behavior of porous shape memory alloys," International Journal of Solids and Structures, vol. 38, no. 48-49, pp. 8653-8671, 2001.

[29] D. C. Lagoudas, Z. Bo, and M. A. Qidwai, "A unified thermodynamic constitutive model for SMA and finite element analysis of active metal matrix composites," Mechanics of Composite Materials and Structures, vol. 3, no. 2, pp. 153-179, 1996.

[30] M. Hakamada, Y. Asao, T. Kuromura, Y. Chen, H. Kusuda, and M. Mabuchi, "Density dependence of the compressive properties of porous copper over a wide density range," Acta Materialia, vol. 55, no. 7, pp. 2291-2299, 2007.

[31] S. P. Timoshenko and J. M. Gere, Theory of Elastic Stability, McGraw-Hill, New York, NY, USA, 1961. 

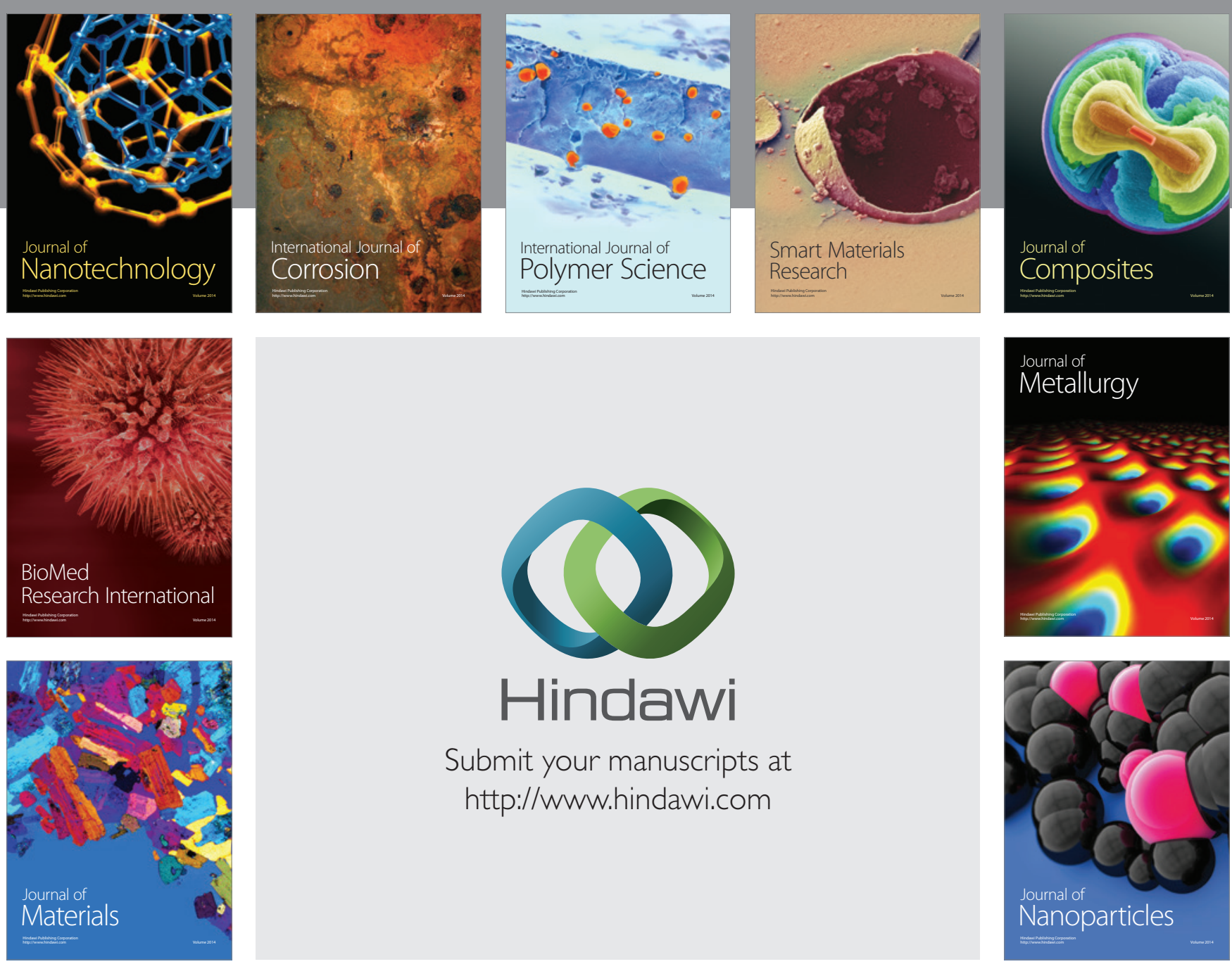

Submit your manuscripts at http://www.hindawi.com
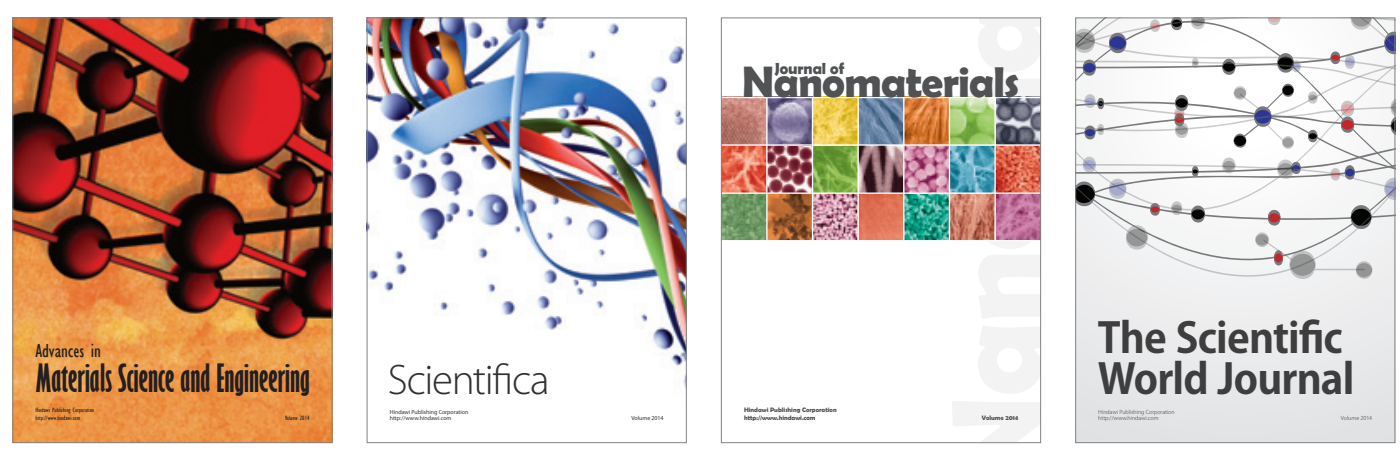

\section{The Scientific World Journal}
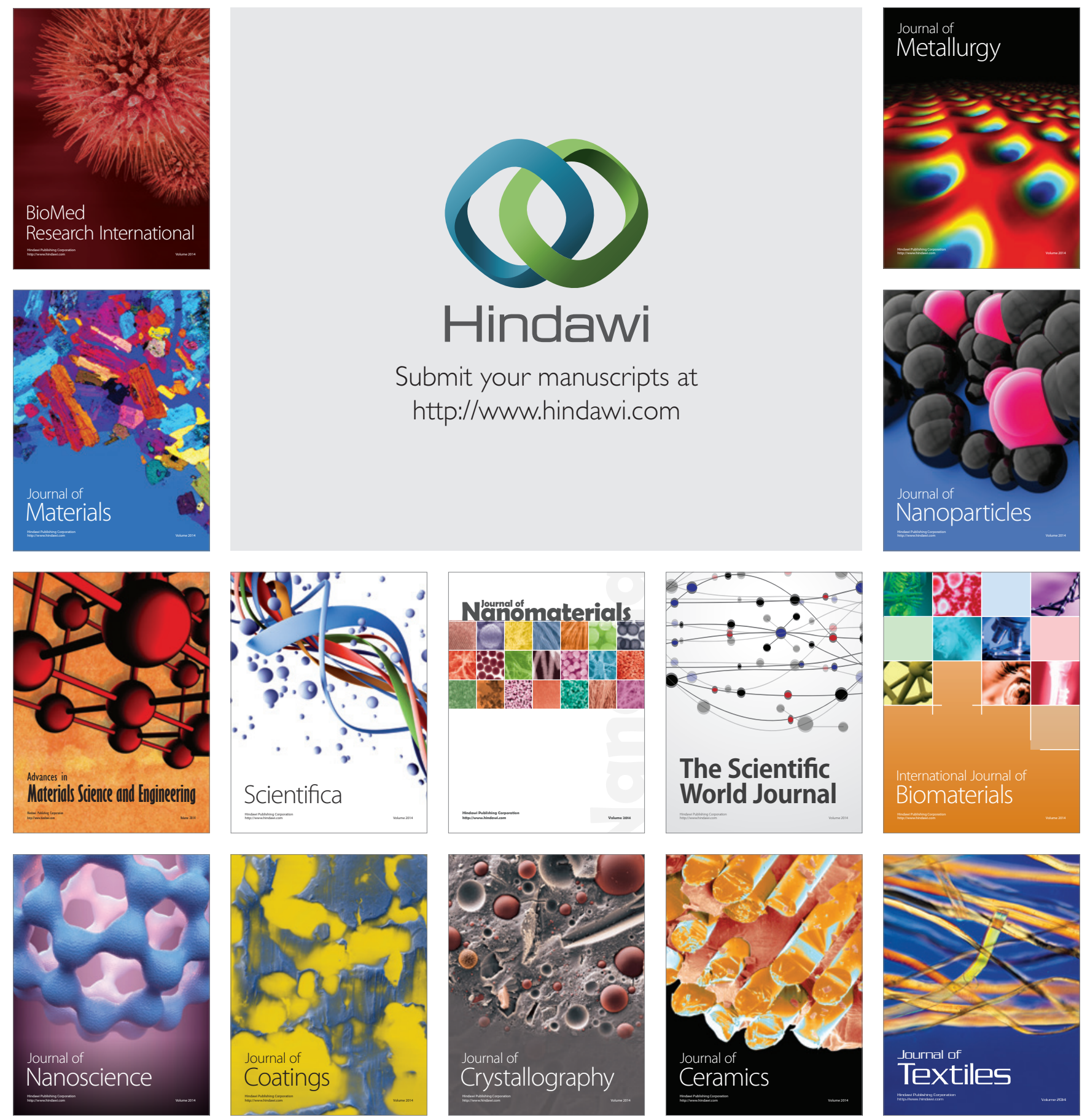